\title{
Entropy production in non-equilibrium fluctuating hydrodynamics
}

\author{
Giacomo Gradenigo, Andrea Puglisi, and Alessandro Sarracino \\ CNR-ISC and Dipartimento di Fisica, Università Sapienza - p.le A. Moro 2, 00185, Roma, Italy
}

Fluctuating entropy production is studied for a set of linearly coupled complex fields. The general result is applied to non-equilibrium fluctuating hydrodynamic equations for coarse-grained fields (density, temperature and velocity), in the framework of model granular fluids. We find that the average entropy production, obtained from the microscopic stochastic description, can be expressed in terms of macroscopic quantities, in analogy with linear non-equilibrium thermodynamics. We consider the specific cases of driven granular fluids with two different kinds of thermostat and the homogeneous cooling regime. In all cases, the average entropy production turns out to be the product of a thermodynamic force and a current: the former depends on the specific energy injection mechanism, the latter takes always the form of a static correlation between fluctuations of density and temperature time-derivative. Both vanish in the elastic limit. The behavior of the entropy production is studied at different length scales and the qualitative differences arising for the different granular models are discussed.

PACS numbers: 05.40.-a,05.70.Ln,45.70.-n 


\section{INTRODUCTION}

Among the several different efforts to describe out-of-equilibrium systems, the linear non-equilibrium thermodynamic approach [1] has obtained many important results, at least for a class of systems close to equilibrium. In this framework, a fundamental role is played by the macroscopic entropy production, which is related to the irreversible phenomena occurring in the system due to the presence of heat and matter currents, chemical reactions, viscous flows, etc... For systems in contact with a single thermal bath, the study of fluctuations relaxing towards the equilibrium state can be described within such a theory, and extensions of it [2]. Moreover, the great interest in the study of entropy production in non-equilibrium stationary states has been motivated by the fact that, in some particular cases, it can be shown that the stationary state is characterized by the minimum entropy production principle [1, 3].

For generally far from equilibrium systems, a comprehensive theory is still lacking, but some important results have been obtained in the last years. In particular, in the framework of systems described by stochastic models, a stochastic thermodynamics has been proposed, extending the concepts of standard thermodynamics to fluctuating quantities, and some general relations have been proved [4-13]. As for classical equilibrium thermodynamics, a central role is played by the microscopic, fluctuating version of entropy production, defined as functional of finite time-length trajectories in the phase space. Such a quantity satisfies relations which have been considered by some authors as generalizations of the second law of thermodynamics [14].

The specific aim of this paper is the study of fluctuations of entropy production in systems described by a set of coupled Langevin equations. Many examples of physical systems are well characterized by such a kind of stochastic process [15 18]. In particular, we are interested in non-equilibrium stationary systems where the coupling among the different fields defining the model, as well as the noise entering the Langevin description, violate the detailed balance condition. In these out-of-equilibrium systems a finite rate of entropy production can be measured. However, from a microscopic point of view, fluctuating entropy production is an elusive quantity: in a non-equilibrium stationary state (NESS) the total entropy $S(t)$ (assuming we have a definition for it) has vanishing time-derivative. In analogy with the perspective of linear non-equilibrium thermodynamics, one can try to separate this zero time-derivative into two opposite contributions: one is a surface term, namely the entropy flow through the boundaries (e.g. heat exchanged with the thermostat) and the other is a bulk, volume term, namely the entropy produced inside the system because of internal lack of detailed balance:

$$
\frac{d S}{d t}=\left.\frac{d S}{d t}\right|_{\text {prod }}+\left.\frac{d S}{d t}\right|_{e x t} .
$$

At equilibrium both terms are vanishing, but in a NESS the production term is always positive. In linear nonequilibrium thermodynamics, symmetry arguments contribute to make this separation unique; however, in general, it is not evident that this unique separation can always be achieved. A definition of the entropy production term related to the dynamics rather than to the thermodynamics of the system has been proposed in recent years:

$$
\left.\frac{d S}{d t}\right|_{\text {prod }}=\lim _{t \rightarrow \infty} \frac{\langle\Sigma(t)\rangle}{t} \geq 0,
$$

where $\Sigma(t)$ is a functional of the trajectory $\{\phi(s)\}$ of the system in the time interval $s \in[0, t]$, with $\phi(s)$ a set of relevant observables. As will be also shown in Sec. IV] $\langle\Sigma\rangle$ is often associated with particular currents (or time-asymmetric correlations) vanishing at equilibrium. Fluctuations of $\Sigma$ on finite-time trajectories in finite-size systems, may display also negative values, with an exponentially small probability, as dictated by the Fluctuation Relations [4, 5].

In this paper we approach the problem on fairly general grounds. Within the framework of Markovian dynamics, we bring to the fore the structural elements which are common to general systems of Langevin equations. In particular, we relate the expressions obtained from the stochastic description of the system to macroscopic quantities, which can be put in a form very similar to that of linear non-equilibrium thermodynamics.

In order to illustrate our general results, we shall focus on the set of non-equilibrium fluctuating hydrodynamic equations for granular fluids [19, 20]. We consider both driven granular gases, where two different kinds of thermostat are studied, and the case of homogeneous cooling [21]. For such systems, in certain ranges of the physical parameters, a description in term of coarse-grained hydrodynamic fields can be given, and a set of coupled Langevin equations can be written, involving density, temperature and velocity fields. Applying our formalism to these systems, we are able to express the average entropy production in terms of macroscopic currents and thermodynamical forces. A similar result has been recently obtained in [22], in the complementary framework of non-equilibrium stationary spin systems, described by transition rates violating detailed balance, and in [12].

The structure of the paper is the following. In Sec. II we briefly recall some concepts of linear non-equilibrium thermodynamics. In Sec. III we obtain an expression for the entropy production of a set of Langevin equations, which is applied to fluctuating hydrodynamic equations for granular fluids in Sec. IV] Finally, some conclusions are drawn in Sec. V. Two Appendices are devoted to some technical details. 


\section{ENTROPY PRODUCTION IN LINEAR NON-EQUILIBRIUM THERMODYNAMICS}

Here we recall some concepts of the linear non-equilibrium thermodynamics theory [1], in order to give a reference frame for the following discussions.

Linear non-equilibrium thermodynamics is a continuum theory which aims at describing macroscopic systems characterized by irreversible processes. The starting point is the balance equation for the entropy, stating that the entropy in a volume changes because entropy flows from the boundaries into the volume, or because some irreversible phenomena are taking place inside the volume. The underlying hypothesis is a "local" equilibrium assumption, which allows one to write the thermodynamic Gibbs relations connecting the local (within a small mass element) entropy with other thermodynamic quantities.

\section{A. Thermodynamic forces and fluxes}

The first step of the theory is the local formulation of the conservation laws. In general, the conservation law for a given conserved quantity $\rho$ (energy, mass, a component of momentum) in the system reads

$$
\frac{\partial \rho(t)}{\partial t}=-\nabla \cdot \mathbf{J}_{\rho}
$$

where $J_{\rho}$ is the flux associated with $\rho$. For an open system, in the presence of sources (or sinks) for the quantity $\rho$, we write the balance equation

$$
\frac{\partial \rho(t)}{\partial t}=-\nabla \cdot \mathbf{J}_{\rho}+\nu_{\rho}
$$

where $\nu_{\rho}$ represents the production (or absorption) of quantity $\rho$ in unit time.

As mentioned above, the variation of entropy $S$ of a macroscopic system is described by a balance equation, namely it can be split in two contributions:

$$
d S=\left.d S\right|_{\text {ext }}+\left.d S\right|_{\text {prod }},
$$

where $\left.d S\right|_{\text {ext }}$ is the entropy change due to the coupling of the system with the surrounding medium, and $\left.d S\right|_{\text {prod }}$ is the entropy produced inside the system due to irreversible processes. For an insulated system, $\left.d S\right|_{\text {ext }}=0$ and then $d S=\left.d S\right|_{\text {prod }} \geq 0$, from the second law of thermodynamics. For closed systems exchanging heat $Q$ with a thermostat at temperature $T$, one has $\left.d S\right|_{\text {ext }}=\frac{d Q}{T}$ and $d S \geq \frac{d Q}{T}$. The change in time of the total entropy $S$ is

$$
\frac{d S}{d t}=\left.\frac{d S}{d t}\right|_{e x t}+\left.\frac{d S}{d t}\right|_{p r o d}=-\int_{A} \mathbf{J}_{\mathbf{s}} \cdot d \mathbf{A}+\int_{V} s d V
$$

where $A$ denotes the contour surface of the system, $\mathbf{J}_{\mathbf{s}}$ is the entropy flow for unit time and unit area, $s$ is the entropy production density per unit time and $V$ the total volume. For open systems, $\mathbf{J}_{\mathbf{s}}$ is the sum of the heat flow divided by the local temperature, $\mathbf{J}_{\mathbf{q}} / T$, plus all the flows of matters from the outside. Using the conservation laws and "local" equilibrium hypothesis, the entropy production can be related to the several different irreversible phenomena occurring inside the system. The structure of the entropy production $s$ is then that of a bilinear form:

$$
s=\sum_{i} \mathbf{J}_{\mathbf{i}} \cdot \mathbf{F}_{\mathbf{i}}
$$

where $\mathbf{J}_{\mathbf{i}}$ are fluxes (or currents) of the quantities describing the system, which are associated with irreversible phenomena, and $\mathbf{F}_{\mathbf{i}}$ are thermodynamic forces (or affinities), related to gradients of state variables (spatial nonhomogeneities) or to external forces. In equilibrium conditions, all the thermodynamic forces vanish and so the entropy production does. Moreover, one also requires that all fluxes vanish with the thermodynamic forces.

Let us consider for instance the case of a metal bar coupled at the edges with two thermostats at different temperatures [12]. In this situation, a stationary temperature profile sets up along the bar, with a surface entropy flux due to flux of heat across the edges and a bulk entropy production due to the sustain of a temperature gradient in the bulk:

$$
s=\frac{1}{T} \mathbf{J}_{\mathbf{q}} \cdot \mathbf{F}_{\mathbf{q}}=-\mathbf{J}_{\mathbf{q}} \cdot \frac{\nabla T}{T^{2}}
$$


where $T$ is the local temperature and the thermodynamic force conjugated to the heat flux is $\mathbf{F}_{\mathbf{q}}=-(\nabla T) / T^{2}$. From the entropy production in the bulk and the knowledge of the geometry of our problem the net flux of entropy across the edges can be then deduced. In Sec. IV] we shall consider homogeneously driven granular systems in two dimensions, where such a simple distinction between surface and bulk contributions cannot be carried out.

\section{B. Onsager coefficients}

In order to close the system of equations for the entropy production, conservation laws and entropy balance equations have to be supplemented by the phenomenological relations, which, as first approximation, express the fluxes in terms of a linear combination of thermodynamic forces

$$
J_{i}=\sum_{j} L_{i j} F_{j}
$$

through the Onsager coefficients $L_{i j}$. Substituting such relations into the expression for entropy production, one obtains

$$
s=\sum_{i, j} L_{i j} F_{i} F_{j}
$$

namely a quadratic expression in the thermodynamic forces. In equilibrium conditions, the thermodynamic forces vanish and, due to Eq. (9), so the fluxes do.

In the physical example introduced above, the phenomenological relation is the Fourier's law

$$
\mathbf{J}_{\mathbf{q}}=-L_{q q} \frac{\nabla T}{T^{2}}=-\lambda \nabla T
$$

where $\lambda$ is the heat conductivity, and

$$
s=L_{q q}\left(\frac{\nabla T}{T^{2}}\right)^{2}
$$

If the forces $F_{i}$ perturb a system at equilibrium, i.e. a system where only conservative (and therefore time-reversible) forces are present, then Onsager has shown that the coefficients $L_{i j}$ satisfy the so-called reciprocal relations [23, 24].

\section{FLUCTUATING ENTROPY PRODUCTION IN STOCHASTIC DYNAMICS}

Statistical systems are conveniently described by stochastic processes. We recall here how the notion of entropy production can be introduced in such a framework. In particular, here we focus on the degree of irreversibility of the dynamics, as measured by the functional $\Sigma\left(\Omega_{0}^{t}\right)$ introduced by Lebowitz and Spohn [7], where $\Omega_{0}^{t}=\{\phi(s)\}$ is a trajectory of the phase space in the time interval $s \in[0, t]$. Let us consider for simplicity a discrete time process, where jumps occur at times $t_{i}$, with $i \in[0, n]$. The functional $\Sigma\left(\Omega_{0}^{t}\right)$ is defined as the ratio between the probability of a given trajectory $P\left(\Omega_{0}^{t}\right)=p\left(\phi_{0}\right) W\left(\phi_{0} \mid \phi_{1}\right) \ldots W\left(\phi_{n-1} \mid \phi_{n}\right)$, where $\phi_{i}=\phi\left(t_{i}\right)$, and the probability of time-reversed trajectory $P\left(\overline{\Omega_{0}^{t}}\right)$ :

$$
\Sigma(t) \equiv \Sigma\left(\Omega_{0}^{t}\right)=\ln \frac{P\left(\Omega_{0}^{t}\right)}{P\left(\overline{\Omega_{0}^{t}}\right)}-\ln \frac{p\left(\phi_{0}\right)}{p\left(\phi_{t}\right)}=\ln \frac{W\left(\phi_{0} \mid \phi_{1}\right) \ldots W\left(\phi_{n-1} \mid \phi_{n}\right)}{W\left(\phi_{n} \mid \phi_{n-1}\right) \ldots W\left(\phi_{1} \mid \phi_{0}\right)}
$$

where $\overline{\Omega_{0}^{t}} \equiv\{\bar{\phi}(t-s)\}$ and $\bar{\phi}=\epsilon \phi$, with $\epsilon= \pm 1$, according to the parity of the field.

In a stationary state, where entropy produced/consumed inside the system is continuously balanced by a flux coming from the boundaries, as in Eq. (6)

$$
0=\frac{d S}{d t}=\left.\frac{d S}{d t}\right|_{e x t}+\left.\frac{d S}{d t}\right|_{p r o d}
$$

we write, following [7], 


$$
\left.\frac{d S}{d t}\right|_{\text {prod }}=\lim _{t \rightarrow \infty} \frac{1}{t}\langle\Sigma(t)\rangle \geq 0
$$

The functional $\Sigma(t)$ depends on a stochastic variable (i.e. a trajectory of a stochastic differential equation) and is itself a stochastic variable with a given probability density: indeed, for finite $t$, it can also take negative values, of course with a probability decreasing with increasing $t$. At large times and for bounded (or negligible) values of the term $\ln \frac{p\left(\phi_{0}\right)}{p\left(\phi_{t}\right)}$, the probability density of $\Sigma$ satisfies the so-called Fluctuation Relation [4, [5, 7, 10, 25, 26].

In the following we will show that, in cases which are relevant for non-equilibrium spatially extended systems, the functional (13) can take a clear thermodynamic meaning, since it can be expressed in terms of macroscopic currents and forces, in strict analogy with the form of entropy production (7) discussed in Sec. IIA.

\section{A. Coupled Langevin equations}

Here we specialize to a continuous space and time Markov process and, hereafter, $\phi$ denotes a complex $d_{\phi^{-}}$ dimensional vector, with components $\phi_{i}\left(i=1, \ldots, d_{\phi}\right)$. The time-reversal transformation on vector $\phi$ is defined as an operator which changes $\phi_{i} \rightarrow \overline{\phi_{i}} \equiv \epsilon_{i} \phi_{i}$ with $\epsilon_{i} \in\{+1,-1\}$ : this implies that both real and imaginary parts of $\phi_{i}$ have the same sign-change upon time-reversal. As shorthand notations we will use $\bar{\phi}$ or $\epsilon \phi$ to indicate the vector made of time-reversed components $\left\{\epsilon_{i} \phi_{i}\right\}$. In a coarse-grained description of a fluid system, instances of even variables are the Fourier components of local density and temperature fluctuations $\left(\epsilon_{i}=+1\right)$, whereas the velocity field is odd $\left(\epsilon_{i}=-1\right)$. We assume that the dynamics of each component of the vector $\phi$ is described by a Langevin equation:

$$
\frac{d \phi_{i}(t)}{d t}=B_{i}[\phi(t)]+\xi_{i}(t)
$$

with $B_{i}$ a complex drift and $\xi_{i}$ a Gaussian process with $\left\langle\xi_{i}(t) \xi_{j}^{*}(s)\right\rangle=2 \delta_{i j} \delta(t-s) D_{i i}$, where the $x^{*}$ denotes the complex conjugate of $x$. If we consider a trajectory between time 0 and time $t$, we can define its time-reversal as $\overline{\phi_{i}(s)}=\epsilon_{i} \phi_{i}(t-s)$. For the complementary discussion starting from the corresponding Fokker-Planck equation, see Appendix B. The stochastic system in Eq. (16) is indeed a good model to describe the behavior of macroscopic variables in many contexts [16]. Often it works as a first approximation, where one retains only the linear part of the dynamics and the effect of nonlinearities is replaced with noise terms.

In order to define a useful projection of the dynamics, let us for the moment neglect the noise terms in Eq. (16), namely $\xi_{i} \equiv 0$. The time-reversal trajectory satisfies the following differential equation:

$$
\frac{d \overline{\phi_{i}(s)}}{d s}=-\epsilon_{i} \frac{d \phi_{i}(t-s)}{d t}=-\epsilon_{i} B_{i}[\phi(t-s)]=-\epsilon_{i} B_{i}[\epsilon \overline{\phi(s)}] .
$$

We notice two particular cases for $B_{i}(\phi)$ :

$$
\begin{aligned}
& B_{i}(\epsilon \phi)=-\epsilon_{i} B_{i}(\phi) \\
& B_{i}(\epsilon \phi)=\epsilon_{i} B_{i}(\phi) .
\end{aligned}
$$

In the first case $\overline{\phi_{i}(s)}$ satisfies exactly the forward equation (16). In the second case it satisfies the same equation with drift changed of sign. Following these two limit cases, we will in general decompose the $i$-th component of the drift as [15]

$$
B_{i}(\phi)=B_{i, r e v}(\phi)+B_{i, i r r}(\phi),
$$

where

$$
\begin{aligned}
B_{i, r e v}(\phi) & =\frac{1}{2}\left[B_{i}(\phi)-\epsilon_{i} B_{i}(\epsilon \phi)\right]=-\epsilon_{i} B_{i, r e v}(\epsilon \phi) \\
B_{i, i r r}(\phi) & =\frac{1}{2}\left[B_{i}(\phi)+\epsilon_{i} B_{i}(\epsilon \phi)\right]=\epsilon_{i} B_{i, i r r}(\epsilon \phi),
\end{aligned}
$$

where $B_{i, r e v}$ and $B_{i, i r r}$ represent the reversible and irreversible contributions to the drift, respectively. 


\section{B. Entropy production functional}

We assume that the system (16) converges to a unique stationary state in the long time limit. Then, let us consider the entropy production in such a stationary state: following the Onsager-Machlup prescription, in the case of an additive Gaussian noise we can write the probability of a trajectory $\Omega_{0}^{t} \equiv\{\phi(s), s=0 \cdots t\}$ as:

$$
P\left(\Omega_{0}^{t}\right)=p[\phi(0)] \exp \left[\mathcal{S}\left(\Omega_{0}^{t}\right)\right], \quad P\left(\overline{\Omega_{0}^{t}}\right)=p[\overline{\phi(0)}] \exp \left[\mathcal{S}\left(\overline{\Omega_{0}^{t}}\right)\right],
$$

where $p(\phi)$ is the weight (probability or density) of state $\phi$ in the steady state, and the action $\mathcal{S}$ is given by 27]

$$
\mathcal{S}\left(\Omega_{0}^{t}\right)=-\int_{0}^{t} d s \sum_{i}^{\prime}\left\{\frac{1}{4 D_{i i, R}}\left[\frac{d \phi_{i}(s)}{d s}-B_{i}[\phi(s)]\right]\left[\frac{d \phi_{i}^{*}(s)}{d s}-B_{i}^{*}[\phi(s)]\right]\right\} .
$$

We have used the notation $\sum_{i}^{\prime}$ to indicate that the sum runs only on those indexes such that $D_{i i} \neq 0[28]$. Due to stationarity, we can always set to zero the initial time of the interval where the entropy production is calculated. Recalling that $\int_{0}^{t} d s f(t-s)=\int_{0}^{t} d s f(s)$, we easily get for the entropy produced by a path $\Omega_{0}^{t}[29]$ :

$$
\Sigma\left(\Omega_{0}^{t}\right)=\ln \frac{P\left(\Omega_{0}^{t}\right)}{P\left(\overline{\Omega_{0}^{t}}\right)}-\ln \frac{p[\phi(0)]}{p[\overline{\phi(t)}]}=\mathcal{S}\left(\Omega_{0}^{t}\right)-\mathcal{S}\left(\bar{\Omega}_{0}^{t}\right)=\int_{0}^{t} d s \sigma(s),
$$

with entropy production rate

$$
\sigma(s)=\sum_{i}^{\prime} \frac{1}{2 D_{i i}} \underbrace{B_{i, i \text { irr }}[\phi(s)]}_{\text {force }} \underbrace{\left(\dot{\phi}_{i}^{*}(s)-B_{i, r e v}^{*}[\phi(s)]\right)}_{\text {current }}+c . c .,
$$

where c.c. denotes the complex conjugate. In this formalism, we can point out how the entropy production is expressed as the product of forces (the drift) multiplied by currents (time derivative of fields). According to the phenomenological relations (9), the irreversible fluxes vanish when the driving forces are switched off. Our identification of forces and fluxes is consistent with such relations: indeed, when $\left\langle B_{i, i r r}[\phi(s)]\right\rangle=0$ we also have $\left\langle\dot{\phi}_{i}^{*}(s)-B_{i, \text { rev }}^{*}[\phi(s)]\right\rangle=0$, as can be seen by averaging equation (16) over the noise. This is in analogy with the macroscopic expressions in linear non-equilibrium thermodynamics.

\section{Linear processes}

We consider now the linear case $B[\phi] \equiv A \phi$ where $A$ is the so-called dynamical matrix:

$$
B_{i}[\phi]=\sum_{j} A_{i j} \phi_{j}=\sum_{j \in \operatorname{rev}(i)} A_{i j} \phi_{j}+\sum_{j \in \operatorname{irr}(i)} A_{i j} \phi_{j}
$$

where the shorthand notation $j \in \operatorname{rev}(i)$ means that the index $j$ runs on the set of indices such that $\epsilon_{j}=-\epsilon_{i}$, while $j \in \operatorname{irr}(i)$ stands for the set of indices such that $\epsilon_{j}=\epsilon_{i}$. In this case one has

$$
\begin{aligned}
\sigma & =\sum_{i}^{\prime} \frac{1}{2 D_{i i}} \underbrace{\left(\sum_{j \in \operatorname{irr}(i)} A_{i j} \phi_{j}\right)}_{\text {force }} \underbrace{\left[\dot{\phi}_{i}^{*}-\sum_{j \in \operatorname{rev}(i)} A_{i j}^{*} \phi_{j}^{*}\right]}_{\text {current }}+\text { c.c. } \\
& =\sum_{i}^{\prime} \frac{1}{2 D_{i i}}\left[\sum_{j \in \operatorname{irr}(i)} A_{i j}\left(\phi_{j} \dot{\phi}_{i}^{*}+\text { c.c. }\right)+\sum_{\substack{j \in \operatorname{irr}(i) \\
l \in \operatorname{rev}(i)}} A_{i j} A_{i l}\left(\phi_{j} \phi_{l}^{*}-\text { c.c. }\right)\right] .
\end{aligned}
$$

In Appendix $\mathrm{A}$ we show that at equilibrium $\langle\sigma\rangle=0$, as expected. Formula (28) is analogous to the macroscopic thermodynamic result (7): it expresses the entropy production as the product of a force by a current. However, in the general case, the formula remains rather abstract, and we need explicit examples to illustrate its meaning. These will be discussed in the following section. 


\section{APPLICATIONS TO GRANULAR FLUIDS}

In this section we show three examples in the framework of granular fluids where, starting from the definition (25), we can obtain an expression for the entropy production similar to Eq. (17).

\section{A. Fluctuating hydrodynamics for driven granular fluids}

We consider a fluid of $N$ identical inelastic hard spheres in dimension $d$, of mass 1 and diameter $r$, in a square box of volume $V$ with external homogeneous stochastic driving. We denote by $\rho$ the packing fraction of the system, which for $d=2$ reads $\rho=N \pi(r / 2)^{2} / V$. The model is defined by giving the equation of motion for the velocity of $i$-th particle:

$$
\dot{v}_{i}(t)=-\gamma_{b} v_{i}(t)+\sqrt{2 T_{b} \gamma_{b}} \zeta_{i}(t)+F_{i}(t)
$$

where $\gamma_{b}$ is a viscous drag, $T_{b}$ is the temperature of the external thermostat, $\zeta_{i}$ is a Gaussian noise with $\left\langle\zeta_{i}\right\rangle=0$ and $\left\langle\zeta_{i}(t) \zeta_{j}\left(t^{\prime}\right)\right\rangle=\delta_{i j} \delta\left(t-t^{\prime}\right)$ and $F_{i}(t)$ is the resulting force of eventual instantaneous collisions with other particles. Every time two particles $i$ and $j$ collide, their velocities are instantaneously changed following the rule

$$
v_{i}^{\prime}=v_{i}-\frac{1+\alpha}{2}\left[\left(v_{i}-v_{j}\right) \cdot \hat{n}\right] \hat{n},
$$

where $\alpha \in[0,1]$ is the restitution coefficient ( $=1$ for elastic collisions) and $\hat{n}$ is the unit vector in the direction joining the centers of the colliding particles. This model has been introduced in [30] and, recently, it has been also successfully used to describe real granular experiments [31, 32]. A different model thermostat is obtained in the limit $\gamma_{b} \rightarrow 0$, $T_{b} \rightarrow \infty$ with constant $\Gamma=2 \gamma_{b} T_{b}$, as it has been studied in [19] (see Sec. IVA3). In both cases the fluid reaches a NESS with a well defined granular temperature $T=\left\langle|v|^{2}\right\rangle / d$. In the case of finite $\gamma_{b}>0$, a characteristic thermostat time $\tau_{b}=1 / \gamma_{b}$ is defined. When compared to the mean collision time $\tau_{c}=1 / \omega$ (with $\omega$ the collision frequency), it determines two possible regimes: $\tau_{b} \gg \tau_{c}$ is the dissipative regime, where $T \leq T_{b}$ (the equal sign holding only for $\alpha=1) ; \tau_{b} \ll \tau_{c}$ is the equilibrium regime where $T=T_{b}$ for any $\alpha$, since collisions are negligible.

Let us add a brief comment on such a model, slightly anticipating some considerations on the entropy production to be discussed below. From Eq. (30), we see that the thermostat is homogeneously coupled to all the particles. Therefore, the energy injection represents by definition a bulk contribution. It is then through collisions, Eq. (31), that the dissipation of energy takes place. Because collisions occur across the whole system, also the energy sink represent a bulk contribution. Therefore, we can imagine that inelastic collisions are perfectly equivalent to a zero temperature reservoir, which is coupled to all the particles. In such a description, what is generally called "entropy flux" refers to the entropy exchanged with reservoirs, rather than to entropy flowing into the system through the boundaries.

In the case where a separation of temporal and spatial scales takes place, a hydrodynamic description of the system can be given. Then, we introduce the coarse-grained hydrodynamic fields, density, velocity and temperature, $n(\mathbf{r}, t), \mathbf{u}(\mathbf{r}, t)$ and $T(\mathbf{r}, t)$, respectively, where $\mathbf{r}$ denotes a point in the $d$-dimensional space, as follows:

$$
\begin{aligned}
n(\mathbf{r}, t) & =\sum_{i} \delta\left(\mathbf{r}-\mathbf{r}_{i}(t)\right), \\
\mathbf{u}(\mathbf{r}, t) & =\frac{1}{n} \sum_{i} \mathbf{v}_{i}(t) \delta\left(\mathbf{r}-\mathbf{r}_{i}(t)\right), \\
T(\mathbf{r}, t) & =\frac{2}{d n} \sum_{i} \frac{v_{i}^{2}(t)}{2} \delta\left(\mathbf{r}-\mathbf{r}_{i}(t)\right) .
\end{aligned}
$$

Here the sums are over all the particles in the system. The homogeneous stationary state is characterized by constant density $n$ and granular temperature $T$. The fluctuations of hydrodynamic fields are $\{\delta n=n(r, t)-n, \delta T=T(r, t)-$ $\left.T, u_{x}, u_{y}\right\}$ and are described by the set of linear hydrodynamic equations. In these equations the small scale fluctuations have been projected out, but their effect on large scale fluctuations can be taken into account by a proper addition of noise terms [16], resulting in a fluctuating hydrodynamic description. For these models, such equations have been studied in [19, 20].

After linearization near the homogeneous state, and changing to Fourier space, in two spatial dimensions $(d=2)$, we are left with a linear Langevin system for the components of a four-dimensional $\left(d_{\phi}=4\right)$ complex field vector $\phi=$ $(\delta n(k), \delta T(k), u(k), v(k))$, with parities under time-reversal $\epsilon=(1,1,-1,-1)$. By $u$ and $v$ we mean the longitudinal 
and transverse velocity field, respectively. In this case a useful shorthand notation consists in replacing the index $i$ by a label equal to the name of the fields (omitting the $\delta$ s for simplicity), i.e. $i=1 \rightarrow n, i=2 \rightarrow T, i=3 \rightarrow u$ and $i=4 \rightarrow v$. The Langevin equation for each component of the vector field is then

$$
\dot{\phi}_{i}=A_{i j} \phi_{j}+\xi_{i}
$$

where the specific form of the dynamic matrix $A$ and of the noise amplitudes depend on the kind of thermostat and will be explicitly given below. In this case, from the definitions following Eq. (27) we have

$$
\begin{aligned}
\operatorname{irr}(n) & =(n, T) \\
\operatorname{irr}(T) & =(n, T) \\
\operatorname{irr}(u) & =(u, v) \\
\operatorname{irr}(v) & =(u, v)
\end{aligned}
$$

$$
\begin{aligned}
& \operatorname{rev}(n)=(u, v) \\
& \operatorname{rev}(T)=(u, v) \\
& \operatorname{rev}(u)=(n, T) \\
& \operatorname{rev}(v)=(n, T),
\end{aligned}
$$

and the set of irreversible and reversible coefficients of the matrix are those with indexes given by, respectively:

$$
\begin{aligned}
\operatorname{irr} & =(n n, n T, T n, T T, u v, u u, v u, v v) \\
\operatorname{rev} & =(n u, n v, T u, T v, u n, u T, v n, v T) .
\end{aligned}
$$

In the following we consider the two kinds of thermostat introduced above.

\section{Finite temperature thermostat, $\gamma_{b}>0$}

In the case $\gamma_{b}>0$, the dynamic matrix reads

$$
A=\left(\begin{array}{cccc}
0 & 0 & 0 & 0 \\
-\gamma_{0} \frac{\omega g_{2} T}{n} & -\left(3 \gamma_{0} \omega+\bar{\kappa} k^{2}+2 \gamma_{b}\right) & 0 & 0 \\
0 & 0 & -\left(\nu_{\|} k^{2}+\gamma_{b}\right) & 0 \\
0 & 0 & 0 & -\left(\nu_{\perp} k^{2}+\gamma_{b}\right)
\end{array}\right)+\mathcal{I}\left(\begin{array}{cccc}
0 & 0 & -k n & 0 \\
0 & 0 & -k \frac{2 p_{0}}{n d} & 0 \\
-k \frac{c^{2}}{n} & -k \frac{p_{0}}{m n T} & 0 & 0 \\
0 & 0 & 0 & 0
\end{array}\right)
$$

where $\nu_{\perp}$ and $\nu_{\|}$are the kinematic shear and longitudinal viscosity respectively, while $\bar{\kappa}=2 \kappa /(n d)$ is the thermal diffusion coefficient associated with the thermal conductivity $\kappa$. Here $\gamma_{0}=\left(1-\alpha^{2}\right) / 2 d$, $g_{2}$ is the pair correlation function at contact (in two dimensions we use the Verlet-Levesque approximation $g_{2}=(1-7 \rho / 16) /(1-\rho)^{2}, p_{0}$ is the pressure, which for elastic hard disks reads $p_{0}=n T\left(1+2 \pi g_{2} n r^{2} / 4\right)$, and $c$ is the thermal velocity. Here and in the following the transport coefficients $\nu_{\perp}, \nu_{\|}$and $\bar{\kappa}$ are evaluated by the Enskog theory for elastic disks [33] at temperature $\mathrm{T}$ (which is the NESS temperature and may depend on inelasticity). Note that irreversible coefficients of $A$ are always real, while reversible coefficients are always imaginary.

Hydrodynamic noise is the sum of external noise due to the bath and internal one due to collisions. Only the latter is assumed to satisfy a "local equilibrium" assumption, i.e. the fluctuation-dissipation relation with the granular temperature [19, 20]. Noises amplitudes have, then, the following amplitudes:

$$
\begin{aligned}
D_{n n} & =0 \\
D_{T T} & =\frac{4 \gamma_{b} T_{b} T}{d n}+\frac{2 k^{2} \bar{\kappa} T^{2}}{n d} \\
D_{u u} & =\frac{\gamma_{b} T_{b}}{n}+\frac{\nu_{\|} k^{2} T}{n} \\
D_{v v} & =\frac{\gamma_{b} T_{b}}{n}+\frac{\nu_{\perp} k^{2} T}{n} .
\end{aligned}
$$

Following the general formula (29), we get - for the entropy production rate at a given $k$ :

$$
\sigma_{k}=\left[\frac{A_{T n}}{D_{T T}}+\frac{i}{k n} \frac{A_{T T} A_{T u}}{D_{T T}}-\frac{i}{k n} \frac{A_{u u} A_{u T}}{D_{u u}}\right] \frac{\delta n \dot{T}^{*}+c . c .}{2}+\text { b.t. }
$$


To obtain the above formula we have used the linearized continuity equation $\dot{\delta n}=-i(k n) u$ which allows one to do the following replacements: $u=i \dot{\delta n} / k n$ and $u^{*}=-i \dot{\delta n}{ }^{*} / k n$. Moreover, we have exploited the fact that all terms of the kind $\phi \dot{\phi}^{*}+$ c.c. are so-called "boundary terms", i.e. they contribute to the entropy produced in the time-interval $[0, t]$ by an amount $|\phi(t)|^{2}-|\phi(0)|^{2}$, which is usually sub-leading with respect to other terms which grow with time $t[25,26$, 34, 35]. We have denoted all these terms by the symbol b.t. (those terms vanish in long-time averages, as dicussed below). Using the definition of the dynamical matrix $A$ in Eq. (45), we have

$$
\sigma_{k}=h(k) \Re\left[\delta n(k) \dot{T}^{*}(k)\right]+\text { b.t. }
$$

where

$$
h(k)=-\frac{\left(T_{b}-T\right)}{n T} \frac{\gamma_{b}\left\{p_{0}\left[\bar{\kappa}+2(3 / d-1) \nu_{\| \mid}\right] k^{2}+g_{2} n\left(\gamma_{b} T_{b}+\nu_{\|} k^{2} T\right)+6 p_{0} \gamma_{b} T_{b} /(d T)\right\}}{\left(\bar{\kappa} k^{2} T+2 \gamma_{b} T_{b}\right)\left(\nu_{\|} k^{2} T+\gamma_{b} T_{b}\right)} .
$$

Here $\Re[x]$ denotes the real part of $x$ and we have used the relation $\gamma_{0} \omega \simeq 2 \gamma_{b}\left(T_{b}-T\right) /(d T)$ [36]. At equilibrium, namely for elastic interactions, $T=T_{b}$ and the entropy production vanishes identically, as expected.

Notice that in the linearized hydrodynamics, modes at different $k$ do not interact and therefore do not exchange energy or produce entropy. Entropy is instead produced separately at each $k$, because of unbalanced fluxes among different components of the fields, i.e. an entropy production rate $\sigma_{k}$ exists for each mode $k$. The total rate of entropy produced by the hydrodynamic degrees of freedom is the sum of all $\sigma_{k}$ in the hydrodynamic range of $k$ s. Interestingly enough, because of the linear approximation adopted, each scale $k$ has its own entropy production which is expected to satisfy the Fluctuation Relation.

\section{Average entropy production}

Eq. (46) is the central result of this paper: it expresses the entropy production defined microscopically in terms of macroscopic quantities, following the structure of linear non-equilibrium thermodynamics. In particular, the quantity $\delta n(k) \dot{T}^{*}(k)$ plays the role of a current of energy, whereas the quantity $h(k)$ is a thermodynamic force which is vanishing at equilibrium. Moreover, taking the stationary average over the stationary distribution on equation (46), the boundary terms vanish, and, exploiting the dynamical equation for the temperature field, the entropy production can be expressed as a linear combination of static correlation functions (structure factors)

$$
\left\langle\sigma_{k}\right\rangle=h(k) \Re\left[\left\langle\delta n(k) \dot{T}^{*}(k)\right\rangle\right]=h(k)\left[A_{T n}^{*} C_{n n}(k)+A_{T T}^{*} C_{n T}(k)+A_{T u}^{*} C_{n u}(k)\right],
$$

where $C_{i j}(k)=\left\langle\phi_{i}(k) \phi_{j}^{*}(k)\right\rangle$. The structure factors can be computed analytically. Then, for small $k$, we have $h(k) \sim a_{0}+a_{2} k^{2}$, with

$$
a_{0}=-\frac{\left(6 p_{0}+d g_{2} n T\right) \gamma_{0} \omega_{0}}{4 n T T_{b} \gamma_{b}}
$$

and $\Re\left[\left\langle\delta n(k) \dot{T}^{*}(k)\right\rangle\right] \sim b_{2} k^{2}$, so that

$$
\left\langle\sigma_{k=0}\right\rangle=0 .
$$

In the opposite limit, $k \rightarrow \infty$, one finds the behavior $\left\langle\sigma_{k}\right\rangle \sim k^{-4}$. In Fig. 1 we show the average entropy production as a function of $k$ for several different values of the parameters $\alpha$ and $\rho$. The first observation is that the average entropy production defined in Eq. (48) vanishes by definition at equilibrium. In this situation, the coefficient $A_{T n}^{*}$ is zero and the correlation functions $C_{n T}(k)$ and $C_{n u}$ between fields of opposite parity under time reversal, vanish due to time reversibility. Moreover, from the results of our calculations we find that, even in inelastic cases, both at very large and very short length scales, the system looks like in equilibrium, where we have a zero entropy production. From the results of a previous work [20], we know that modes at $k \rightarrow 0$ show equipartition at the bath temperature $T_{b}$, whereas modes for $k$ very large at the granular temperature $T$. It makes sense that at the length-scales where a good degree of equipartition is attained the entropy production turns to be zero. Another interesting similarity with the results on fluctuating hydrodynamics obtained in [20] is that, also here, from the study of the entropy production, a characteristic non-equilibrium length-scale emerges. It seems that there is a preferential wave-vector at which the excitation of modes in the system produces the higher degree of dissipation, and that this wave-vector corresponds to a finite length. 


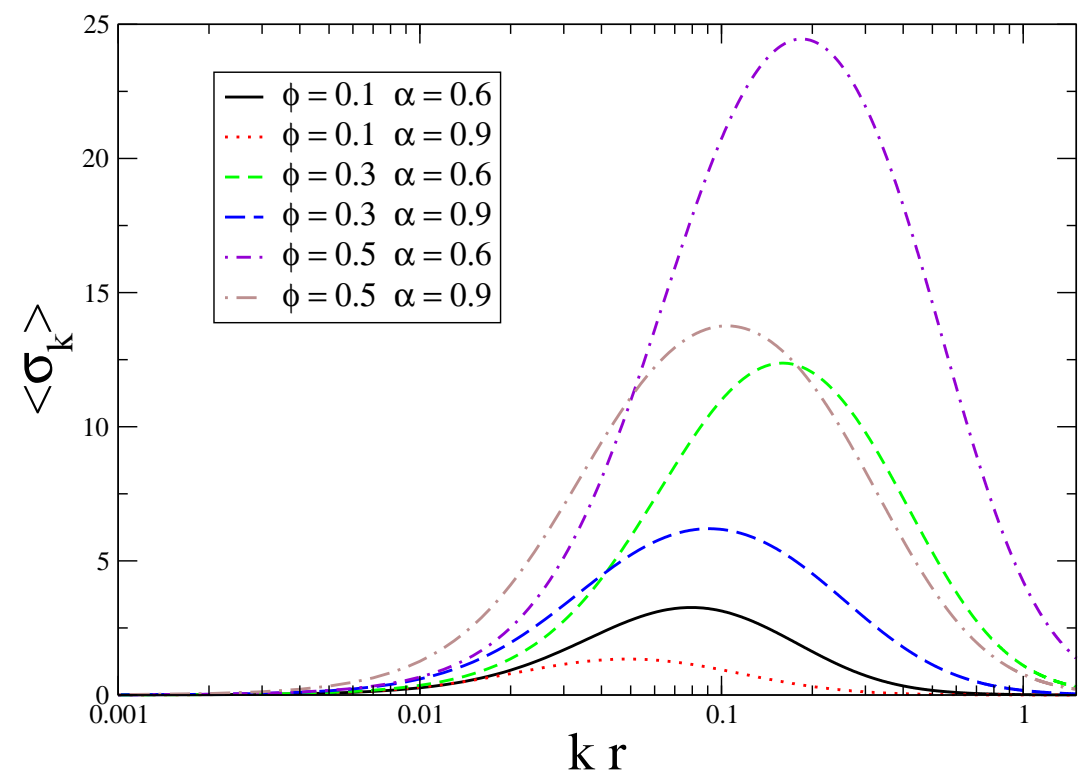

FIG. 1. Average entropy production $\left\langle\sigma_{k}\right\rangle$ as a function of $k r$, Eq. [46), for the driven granular model with finite temperature thermostat and friction $\gamma_{b}>0$ (defined by the Eqs. (33) and (40.44)). The different curves show the behavior of $\left\langle\sigma_{k}\right\rangle$ for several values of the parameters $\alpha$ and $\rho$, as reported in the legend. Notice the peak at a certain wave-vector which signals the presence of a characteristic length-scale, as explained in the text.

\section{Infinite temperature thermostat}

For the case $\gamma_{b}=0$ and $T_{b} \rightarrow \infty$ with finite $\Gamma=2 \gamma_{b} T_{b}$, the dynamical matrix reads

$$
A=\left(\begin{array}{cccc}
0 & 0 & 0 & 0 \\
-\gamma_{0} \frac{\omega g_{2} T}{n} & -\left(3 \gamma_{0} \omega+\bar{\kappa} k^{2}\right) & 0 & 0 \\
0 & 0 & -\nu_{\|} k^{2} & 0 \\
0 & 0 & 0 & -\nu_{\perp} k^{2}
\end{array}\right)+\mathcal{I}\left(\begin{array}{cccc}
0 & 0 & -k n & 0 \\
0 & 0 & -k \frac{2 p_{0}}{n d} & 0 \\
-k \frac{c^{2}}{n} & -k \frac{p_{0}}{m n T} & 0 & 0 \\
0 & 0 & 0 & 0
\end{array}\right)
$$

and noise amplitudes are

$$
\begin{aligned}
D_{n n} & =0 \\
D_{T T} & =\frac{1}{2}\left(\frac{4 T \Gamma}{d n}+\frac{4 k^{2} \bar{\kappa} T^{2}}{n d}\right) \\
D_{u u} & =\frac{1}{2}\left(\frac{\Gamma}{n}+\frac{2 \nu_{\|} k^{2} T}{n}\right) \\
D_{v v} & =\frac{1}{2}\left(\frac{\Gamma}{n}+\frac{2 \nu_{\perp} k^{2} T}{n}\right) .
\end{aligned}
$$

Then, following exactly the same computation as before, we find

$$
\left\langle\sigma_{k}\right\rangle=h^{\prime}(k) \Re\left[\left\langle\delta n(k) \dot{T}^{*}(k)\right\rangle\right]
$$

with

$$
h^{\prime}(k)=-\frac{\gamma_{0} \omega_{0}\left\{\left[2 p_{0}\left(\bar{\kappa}+\nu_{||}\right)+d g_{2} n T \nu_{\mid]}\right] k^{2}+\left(6 p_{0}+d g_{2} n T\right) \gamma_{0} \omega_{0}\right\}}{2 n T_{g}^{2}\left(\bar{\kappa} k^{2}+2 \gamma_{0} \omega_{0}\right)\left(\nu_{\|} k^{2}+\gamma_{0} \omega_{0}\right)} .
$$

In this case, the series expansion of the prefactor $h^{\prime}(k)$, for small $k$, gives $h^{\prime}(k) \sim a_{0}^{\prime}+a_{2}^{\prime} k^{2}$, with 


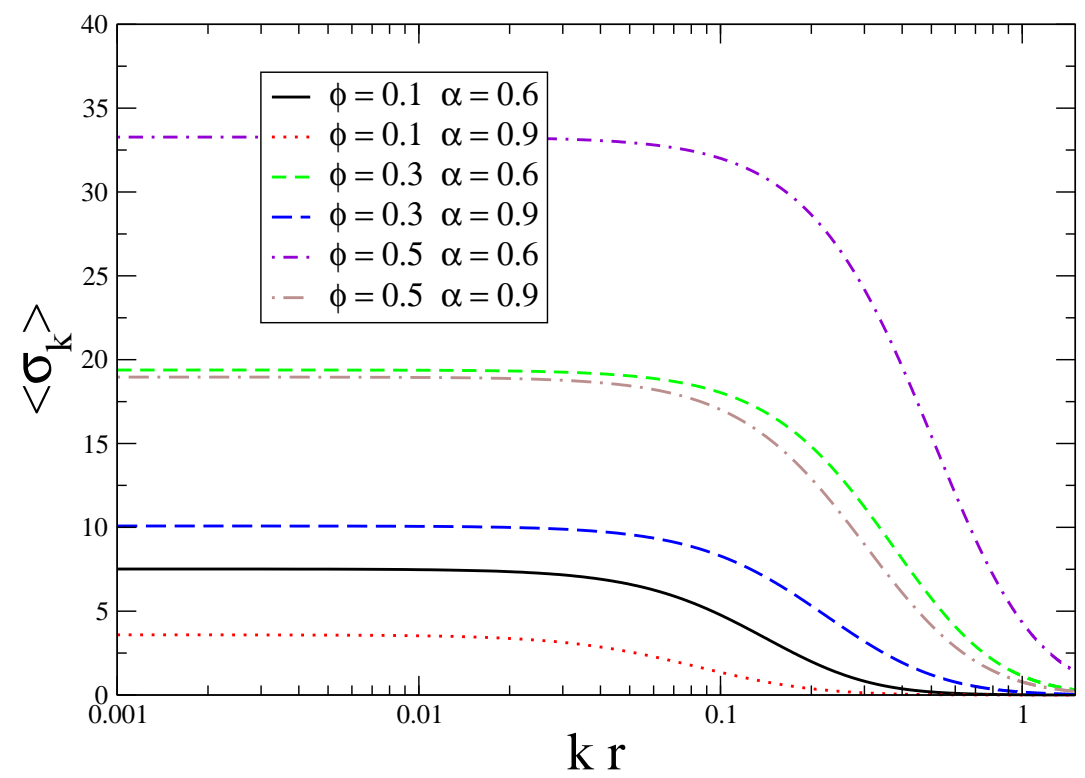

FIG. 2. Average entropy production $\left\langle\sigma_{k}\right\rangle$ as a function of $k r$, Eq. (56), for the driven granular model with infinite temperature thermostat and without friction, $T_{b}=\infty, \gamma_{b}=0$ with $2 \gamma_{b} T_{b}=\Gamma$ finite, defined by the Eqs. (33) and (51.55). The different curves show the behavior of $\left\langle\sigma_{k}\right\rangle$ for several values of the parameters $\alpha$ and $\rho$, as reported in the legend. Notice that $\sigma_{k}$ reaches a finite value for $k \rightarrow 0$.

$$
a_{0}^{\prime}=-\frac{6 p_{0}+d g_{2} n T}{4 n T^{2}}
$$

while $\left\langle\Re\left[\delta n(k) \dot{T}^{*}(k)\right]\right\rangle \sim b_{0}^{\prime}+b_{2}^{\prime} k^{2}$, with

$$
b_{0}^{\prime}=-\frac{n T^{2}\left(6 p_{0}+d g_{2} n T\right) \gamma_{0} \omega_{0}}{6 p_{0}^{2}+d g_{2} n p_{0} T+9 d n^{2} T \gamma_{0} \nu_{\|} \omega_{0}},
$$

so that for the entropy production at $k=0$ we have the finite value

$$
\left\langle\sigma_{k=0}\right\rangle=\frac{\left(6 p_{0}+d g_{2} n T\right)^{2} \gamma_{0} \omega_{0}}{4\left(6 p_{0}^{2}+d g_{2} n p_{0} T+9 d n^{2} T \gamma_{0} \nu_{\|} \omega_{0}\right)} .
$$

This result is qualitatively different from that obtained in the case of a finite temperature thermostat. The finite value for $k \rightarrow 0$ obtained in this case could be related to the scale-free correlations in the fluctuations of the hydrodynamic fields of this model [37]. In the opposite limit, $k \rightarrow \infty$, we again find $\left\langle\sigma_{k}\right\rangle \sim k^{-4}$. In Fig. 2 we show the average entropy production as a function of $k$ for several different values of the parameters $\alpha$ and $\rho$. Notice here that there is always a finite entropy production, even at very short wave-vectors, where a good degree of equipartition between modes is never obtained. The result we find is consistent with the fact that it does not exist any finite length-scale at which we can coarse grain the local hydrodynamics fields in order to see an equilibrium-like behavior.

\section{B. Homogeneous cooling}

In the limit $\Gamma \rightarrow 0$, the above model becomes a pure cooling granular fluid (no energy injection). If the initial state is spatially homogeneous, a regime exists where homogeneity is preserved [38], the temperature decays following the Haff's law and any time-dependence is enslaved by the temperature. This regime, known as Homogeneous Cooling State (HCS) lasts until instabilities arise and homogeneity is broken. In the HCS, it is useful to introduce a new timescale, defined by $d \tau=\omega(t) d t$ where $\omega(t)$ is the time-dependent collision frequency and, at the same time, rescaling 
density, velocity and temperature fluctuations by $n$ (which is constant), $\sqrt{2 T(t)}$ and $T(t)$ respectively. With such prescriptions one achieves a stationary representation of the HCS whose Fourier-transformed linearized hydrodynamics has been studied for example in [21, 39]. In this case, the dynamical matrix reads

$$
A=\left(\begin{array}{cccc}
0 & 0 & 0 & 0 \\
-\gamma_{0} g_{2} & -\left(\gamma_{0}+\tilde{\bar{\kappa}} k^{2}\right) & 0 & 0 \\
0 & 0 & \gamma_{0}-\tilde{\nu_{\|}} k^{2} & 0 \\
0 & 0 & 0 & \gamma_{0}-\tilde{\nu_{\perp}} k^{2}
\end{array}\right)+\mathcal{I}\left(\begin{array}{cccc}
0 & 0 & -k \lambda_{0} & 0 \\
0 & 0 & -k \frac{2 p_{0}}{d n T} \lambda_{0} & 0 \\
-k c^{2} \lambda_{0} & -k \frac{p_{0}}{2 n T} \lambda_{0} & 0 & 0 \\
0 & 0 & 0 & 0
\end{array}\right)
$$

and noise amplitudes are

$$
\begin{aligned}
D_{n n} & =0 \\
D_{T T} & =\frac{1}{2} \frac{4 k^{2} \bar{\kappa}}{n d \omega} \\
D_{u u} & =\frac{1}{2} \frac{\nu_{\|} k^{2}}{n \omega} \\
D_{v v} & =\frac{1}{2} \frac{\nu_{\perp} k^{2}}{n \omega} .
\end{aligned}
$$

Here $\tilde{\bar{\kappa}}=\bar{\kappa} / \omega, \tilde{\nu_{\|}}=\nu_{\|} / \omega$ and $\tilde{\nu_{\perp}}=\nu_{\perp} / \omega$ are the rescaled transport coefficients, $\lambda_{0}$ is the mean free path and $c^{2}=1 /(2 T)(\partial p / \partial n)_{T}$. Note that the above matrices are obtained by replacing $\gamma_{b} \rightarrow-\gamma_{0} \omega$ in the matrices for the driven case (and of course applying the other rescalings discussed above). Note also that the real part of some of the eigenvalues of the hydrodynamical matrix become positive for wave-vectors smaller than the cut-off value $k^{*}$ : such an instability, which marks the end of the HCS, can be prevented by taking a system small enough. Considering values of $k<k^{*}$, for the HCS we find the following expression of the entropy production

$$
\left\langle\sigma_{k}\right\rangle=h^{\prime \prime}(k) \Re\left[\delta n(k) \dot{T}^{*}(k)\right]
$$

with

$$
h^{\prime \prime}(k)=-\frac{d \gamma_{0} \omega\left[g_{2} \nu_{\|}+\frac{2 p_{0}}{d n T} \lambda_{0}\left(\bar{\kappa}+\nu_{\|}\right)\right]}{2 \bar{\kappa} k^{2} \nu_{\|}} .
$$

In Fig. 3 we show the behavior of the average entropy production as a function of $k / k^{*}$, for different values of $\alpha$ and $\rho$. Notice that, even approaching the value $k^{*}$, where instabilities arise, the entropy production remains finite. Also in this case, in the limit $k \rightarrow \infty$ we have the behavior $\left\langle\sigma_{k}\right\rangle \sim k^{-4}$.

\section{CONCLUSIONS}

The coarse-grained dynamics of spatially extended systems can often be described by a set of coupled Langevin equations. For this class of stochastic systems, we have presented a general formula for the fluctuating entropy production, showing that it can be put in a form similar to that expected from macroscopic theories, such as linear non-equilibrium thermodynamics. As an application, we have studied in detail the fluctuating hydrodynamic equations describing the large scales fluctuations of different kinds of granular fluids. In particular, we have singled out that, in homogeneously driven granular fluids, the continuous flow of energy across the system produces some correlations $\langle\delta n \dot{T}\rangle$, usually absent at equilibrium. Namely, a density fluctuation in a certain point of space couples to the variations in time of the local temperature in the neighborhood. We identified the quantity $\delta n \dot{T}$ with the out-of-equilibrium fluctuating current of such a system. Notice that, due to the homogeneous driving mechanism, this current has no direction in space (only in time), differently from many examples of off-equilibrium systems. Moreover, we have shown that the wave-vector dependent entropy production, $\sigma_{k}$, is a quantity that provides a measure of how much dissipative is the granular system when variables are coarse-grained on a length-scale $\lambda \sim 1 / k$.

\section{ACKNOWLEDGMENTS}

We thank E. Trizac and D. Villamaina for useful discussions. The work is supported by the "Granular-Chaos" project, funded by the Italian MIUR under the FIRB-IDEAS grant number RBID08Z9JE. 


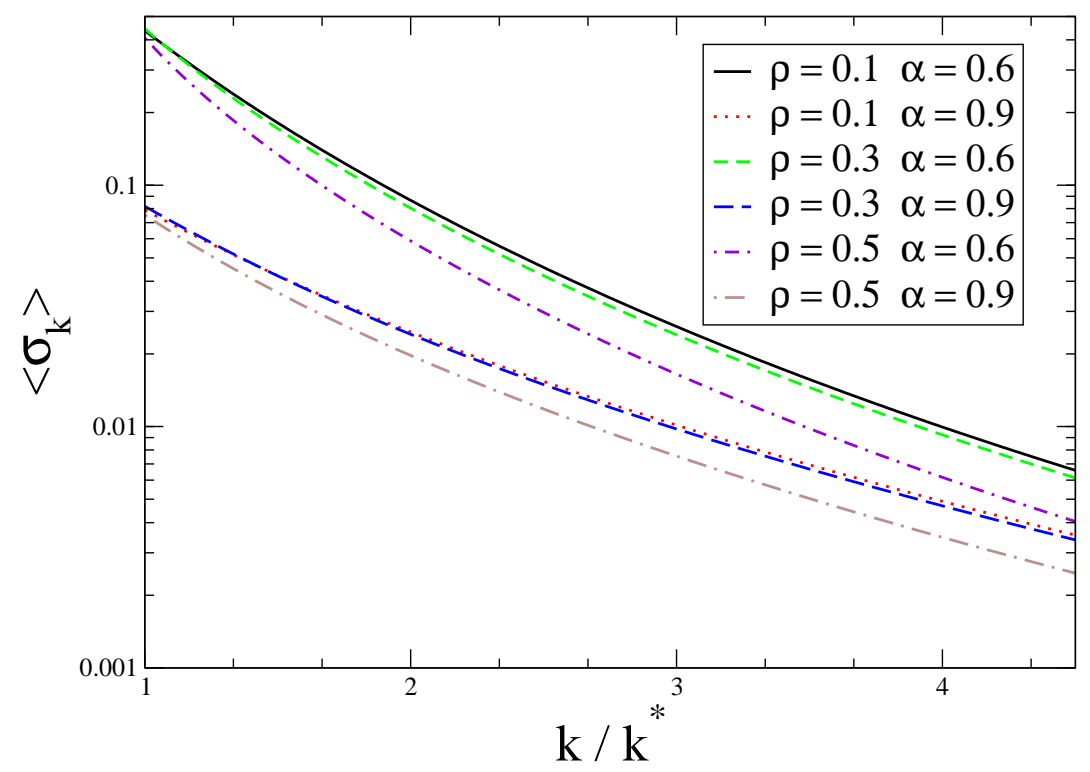

FIG. 3. Average entropy production $\left\langle\sigma_{k}\right\rangle$ as a function of $k / k^{*}$, Eq. (66), for the homogeneous cooling state, defined by the Eqs. (33) and (61,65). The different curves show the behavior of $\left\langle\sigma_{k}\right\rangle$ for several values of the parameters $\alpha$ and $\rho$, as reported in the legend. Notice that $\sigma_{k}$ reaches a finite value for $k=k^{*}$, where $k^{*}$ is the wave-vector of the instability in the system.

\section{Appendix A}

In this Appendix we show that the entropy production vanishes identically when equilibrium conditions are considered. In the following sum over repeated indices is always meant.

Our purpose here is to skecth the main idea, and this is best done - for the sake of compactness and without losing in generality - restricting to the case of all real fields. For such a case the entropy production is given by

$$
\begin{aligned}
\sigma^{\prime}(t) & \equiv \sigma(t)+\ln p(\phi(0))-\ln p(\phi(t))=D_{k k}^{-1} \int_{0}^{t} d s B_{k, i r r}(s)\left[\dot{\phi}_{k}(s)-B_{k, r e v}(s)\right]+\ln p(\phi(0))-\ln p(\phi(t)) \\
& =D_{k k}^{-1} \int_{0}^{t} d s\left(A^{i r r}\right)_{k j} \phi_{j}(s) \dot{\phi}_{k}(s)-D_{k k}^{-1} \int_{0}^{t} d s\left(A^{i r r}\right)_{k j} \phi_{j}(s)\left(A^{r e v}\right)_{k l} \phi_{l}(s)+\ln p(\phi(0))-\ln p(\phi(t)) \\
& =\frac{1}{2} D_{k k}^{-1}\left(A^{i r r}\right)_{k j}\left[\phi_{j}(t) \phi_{k}(t)-\phi_{j}(0) \phi_{k}(0)\right]-D_{k k}^{-1} \int_{0}^{t} d s\left(A^{i r r}\right)_{k j} \phi_{j}\left(A^{r e v}\right)_{k l} \phi_{l}+\ln p(\phi(0))-\ln p(\phi(t)),
\end{aligned}
$$

where, following the definitions (21|22), $\left(A^{i r r}\right)_{i j}=1 / 2\left(A_{i j}+\epsilon_{i} A_{i j} \epsilon_{j}\right)$ and $\left(A^{r e v}\right)_{i j}=1 / 2\left(A_{i j}-\epsilon_{i} A_{i j} \epsilon_{j}\right)$. Using the equilibrium distribution

$$
p(\phi) \propto \exp \left[-\frac{1}{2} \phi_{k}\left(C^{-1}\right)_{k l} \phi_{l}\right]
$$

together with the equilibrium relation

$$
A^{i r r} C=-D \Rightarrow D^{-1} A^{i r r}=-C^{-1},
$$

one immediately finds that, in Eq. (A1), the first term on the rhs of the last line exactly cancels the quantity $\ln p(\phi(0))-\ln p(\phi(t))$. The remaining term can be recast in the form

$$
D_{k k}^{-1}\left(A^{i r r}\right)_{k j} \phi_{j}\left(A^{r e v}\right)_{k l} \phi_{l}=-\left(C^{-1}\right)_{k j} \phi_{j}\left(A^{r e v}\right)_{k l} \phi_{l}=-\left(C^{-1}\right)_{j k} \phi_{j}\left(A^{r e v}\right)_{k l} \phi_{l}=-\phi C^{-1} A^{r e v} \phi,
$$


where the first equality follows from the relation (A3), while the property $C=C^{T}$ has been used in the second. Now, since at equilibrium

$$
A^{r e v} C+C\left(A^{r e v}\right)^{T}=0 \Rightarrow C^{-1} A^{r e v}+\left(C^{-1} A^{r e v}\right)^{T}=0,
$$

one has that $C^{-1} A^{r e v}$ is antisymmetric, namely

$$
\phi C^{-1} A^{r e v} \phi=0 .
$$

This implies $\sigma^{\prime}=0$.

For the full complex case, the demonstration follows in a similar way.

\section{Appendix B}

In this appendix we present some complementary considerations in terms of the Fokker-Planck equation associated with the Langevin equations (16). This allows us to discuss relations involving the Onsager coefficients.

The Fokker-Planck equation obeyed by the probability density for a linear process defined by Eqs. (16) and (27) reads

$$
\frac{d p(\phi, t)}{d t}=-\sum_{i} \frac{\partial}{\partial \phi_{i}}\left[j_{i}(\phi, t) p(\phi, t)\right]
$$

with

$$
j(\phi, t)=-A \phi-R f(\phi, t) .
$$

Here $j$ is the probability current vector, $A$ the dynamical matrix,

$$
f_{i}(\phi, t)=\frac{\partial \ln p(\phi, t)}{\partial \phi_{i}},
$$

and $R$ is any matrix which satisfies $R^{s}=D$ with $R^{s}$ its symmetrized and $D$ the matrix of noise correlations (which is always symmetric by definition). This arbitrariety on $R$ will be discussed below. We anticipate that, at equilibrium, $R$ coincides with the matrix of the Onsager coefficients $L$.

The stationary solution of Eq. (B1), $p(\phi)=\lim _{t \rightarrow \infty} p(\phi, t)$, satisfies

$$
\ln [p(\phi)]=\text { const. }-\frac{1}{2} \phi G \phi^{\dagger},
$$

where $\phi^{\dagger}$ is the adjoint of the vector $\phi$ (transpose and conjugate) and $G=C^{-1}$ with $C_{i j}=\left\langle\phi_{i} \phi_{j}^{*}\right\rangle$ the self-adjoint matrix of covariances. Let us also define the quantity

$$
f^{0}(\phi)=\lim _{t \rightarrow \infty} f(\phi, t)=-G \phi
$$

By direct substitution one finds that $C$ must satisfy the following equation:

$$
-D=[A C]^{s} \equiv \frac{A C+C A^{\dagger}}{2} .
$$

In general this means that

$$
A C=R+Q
$$

with $Q$ an antihermitian matrix, i.e. $Q=-Q^{\dagger}$. The relation with the $D$ matrix is also ambiguous, i.e. $A C=D+Q^{\prime}$, with $Q^{\prime}$ another (in principle different from $Q$ ) antihermitian matrix. In the stationary state one has, for the probability current:

$$
j^{0}(\phi)=\lim _{t \rightarrow \infty} j(t)=-(R G) \phi-(Q G) \phi+(R G) \phi=-(Q G) \phi
$$


Therefore, the probability current at any time may be rewritten as

$$
j(\phi, t)=-R\left[f(\phi, t)-f^{0}(\phi)\right]+j^{0}(\phi) .
$$

The equilibrium condition is $j^{0}(\phi)=0$, which requires $Q=0$, leading to $R=A C$, which is the fluctuationdissipation relation (FDR). Moreover, at equilibrium, from the time translation and time inversion invariance of the joint distribution $p(\phi(t), t ; \phi(0), 0)=p(\overline{\phi(0)}, t ; \overline{\phi(t)}, 0)$, there follow the relations

$$
\begin{aligned}
& C_{i j}=\epsilon_{i} \epsilon_{j} C_{j i}^{*} \\
& L_{i j}=\epsilon_{i} \epsilon_{j} L_{j i}^{*} .
\end{aligned}
$$

On the other hand, a non-equilibrium stationary state with $j^{0} \neq 0$ can be observed for open systems where energy or matter is exchanged with the boundaries/thermostats with a preferred direction in time: this is the case of the driven granular fluids discussed in Section IV

Finally, note that in the Fokker-Planck equation, which is uniquely determined by the linear Langevin equation, only $A$ and $D=R^{s}$ are fixed (and consequently also $C$ or $G$ ). The matrix $R$ is determined only in its hermitian part, while its antihermitian part is undetermined. At equilibrium this indeterminacy disappears, because of the FDR which fixes $R=A C$. Note also that the FDR does not imply $R$ (or $A C$ ) to be symmetric. Its symmetry properties are determined by the parities under time-reversal (B11), as discovered by Onsager [23, 24]: $R_{i j}=R_{j i}^{*} \epsilon_{i} \epsilon_{j}$. Moreover, let us remark that the Onsager matrix is uniquely defined only at equilibrium. Indeed one has at least two options to define the Onsager matrix in the non-equilibrium case: 1) one can follow the principle that the Onsager matrix is the "proportionality factor" between thermodynamic force $f-f^{0}$ and the current excess with respect to $j^{0}$, so that Eq. (B9) implies $L \equiv R ; 2$ ) one can follow the principle that the Onsager matrix is the proportionality factor between the decay of fluctuations and the stationary entropic force $X=-f^{0}$, i.e. $d\langle\phi\rangle / d t=-L\langle X\rangle$, and this implies $L \equiv A C$. At equilibrium the two options coincide. Of course those two principles are still arbitrary: Onsager matrix is, indeed, undefined in non-equilibrium dynamics.

[1] S. R. de Groot and P. Mazur, Non-equilibrium thermodynamics (Dover Publications, New York, 1984).

[2] J. M. Rubí and P. Mazur, Physica A 276, 477 (2000).

[3] A. Pérez-Madrid, J. Chem. Phys. 123, 204108 (2005).

[4] D. J. Evans, E. G. D. Cohen, and G. P. Morriss, Phys. Rev. Lett. 71, 2401 (1993).

[5] G. Gallavotti and E. G. D. Cohen, J. Stat. Phys. 80, 931 (1995).

[6] J. Kurchan, J. Phys. A 31, 3719 (1998).

[7] J. L. Lebowitz and H. Spohn, J. Stat. Phys. 95, 333 (1999).

[8] C. Jarzynski, Phys. Rev. Lett. 78, 2690 (1997).

[9] G. E. Crooks, Phys. Rev. E 60, 2721 (1999).

[10] U. Seifert, Phys. Rev. Lett. 95, 040602 (2005).

[11] T. Hatano and S. Sasa, Phys. Rev. Lett. 86, 3463 (2001).

[12] R. E. Spinney and I. J. Ford, arXiv:1203.0485 (2012).

[13] T. Rao, T. Xiao, and Z. Hou, J. Chem. Phys. 134, 214112 (2011).

[14] J. Kurchan, J. Stat. Mech. , P07005 (2007).

[15] H. Risken, The Fokker-Planck equation: Methods of solution and applications (Springer-Verlag, Berlin, 1989).

[16] J. M. O. de Zarate and J. V. Sengers, Hydrodynamic fluctuations in fluids and fluid mixtures (Elsevier, 2006).

[17] G. H. Fredrickson, J. Chem. Phys. 117, 6810 (2002).

[18] R. Hegger and G. Stock, J. Chem. Phys. 130, 034106 (2009).

[19] T. P. C. van Noije, M. H. Ernst, E. Trizac, and I. Pagonabarraga, Phys. Rev. E 59, 4326 (1999).

[20] G. Gradenigo, A. Sarracino, D. Villamaina, and A. Puglisi, J. Stat. Mech. , P08017 (2011).

[21] J. J. Brey, J. W. Dufty, C. S. Kim, and A. Santos, Phys. Rev. E 58, 4638 (1998).

[22] M. J. de Oliveira, J. Stat. Mech. , P12012 (2011).

[23] L. Onsager, Phys. Rev. 37, 405 (1931).

[24] L. Onsager, Phys. Rev. 38, 2265 (1931).

[25] R. van Zon and E. G. D. Cohen, Phys. Rev. Lett. 91, 110601 (2003).

[26] A. Puglisi, L. Rondoni, and A. Vulpiani, J. Stat. Mech. , P08010 (2006).

[27] L. Onsager and S. Machlup, Phys. Rev. 91, 1505 (1953).

[28] S. Machlup and L. Onsager, Phys. Rev. 91, 1512 (1953).

[29] A. Puglisi and D. Villamaina, Europhys. Lett. 88, 30004 (2009).

[30] A. Puglisi, V. Loreto, U. M. B. Marconi, A. Petri, and A. Vulpiani, Phys. Rev. Lett. 81, 3848 (1998). 
[31] G. Gradenigo, A. Sarracino, D. Villamaina, and A. Puglisi, Europhys. Lett. 96, 14004 (2011).

[32] A. Puglisi, A. Gnoli, G. Gradenigo, A. Sarracino, and D. Villamaina, J. Chem. Phys. 136, 014704 (2012).

[33] S. Chapman and T. G. Cowling, The Mathematical Theory of Non-uniform Gases (Cambridge University Press, 1970).

[34] R. J. Harris, A. Rákos, and M. Schütz, Europhys. Lett. 75, 227 (2006).

[35] F. Bonetto, G. Gallavotti, A. Giuliani, and F. Zamponi, J. Stat. Phys. 123, 39 (2006).

[36] A. Sarracino, D. Villamaina, G. Costantini, and A. Puglisi, J. Stat. Mech. , P04013 (2010).

[37] E. Trizac, I. Pagonabarraga, T. P. C. van Noije, and M. H. Ernst, Phys. Rev. E 65, 011303 (2001).

[38] J. W. Dufty, Journal of Physics: Condensed Matter 12, A47 (2000).

[39] T. P. C. van Noije and M. H. Ernst, Phys. Rev. E 61, 1765 (2000). 